\title{
SPECIAL TECHNOLOGIES AND NEW DEVELOPMENTS TO IMPROVE SLAB QUALITY*
}

\author{
Christian Geerkens ${ }^{1}$ \\ Jochen Wans ${ }^{2}$ \\ Dirk Lieftucht ${ }^{3}$ \\ Artemy Krasilnikov ${ }^{4}$ \\ Martin Klein ${ }^{5}$
}

\begin{abstract}
Increasing requirements on slab quality initiates SMS group in developing products with a high sophisticated level regarding production and process. These characteristics are represented by SMS group's HD family. HD stands for High Definition or the improvement of product quality via higher precision of the casting processes. A deep look inside into casting process is realized by the use of fiber optical equipped HD mold. High definition casting process control is assured using the HD spray and HD size. For objective, environmentally friendly and highly informative analyze of the inner quality of the cast products the new HD scan is present.
\end{abstract}

Keywords: Breakout prediction; Fiber optic sensor; Ultrasonic sensor; Etching.

1 Dipl.-Ing., Executive Vice President, Metallurgical Plants and Environmental Technology Division, SMS Siemag AG, Düsseldorf, Germany.

2 Dr., General Manager, Technical Sales Special Technologies / New Developments, SMS Siemag AG, Düsseldorf, Germany.

3 Dr., Deputy General Manager, Components Development, SMS Siemag AG, Düsseldorf, Germany.

4 Dipl.-Ing., Project Manager, Components Development, SMS Siemag AG, Düsseldorf, Germany.

5 Dipl.-Ing., Project Manager, Technical Sales Special Technologies / New Developments, SMS Siemag AG, Düsseldorf, Germany. 


\section{INTRODUCTION}

The HD mold reproduces thanks to fiber sensor technology solidification process in high resolution. With HD mold steel producers are able to reduce product defects and control the slab quality. Some steel grades, especially the production of different slab widths require highest flexibility on secondary cooling. SMS group introduces a secondary cooling concept HD spray with sensitive edge cooling. The HD size precisely measures the strand width for an early process stage adjustment of the mold width. The analysis of the inner slab quality is realized by the highly informative environmentally friendly HD scan using ultrasonic testing procedure.

\section{HD MOLD FIBER OPTICAL MOLD MONITORING SYSTEM}

The measuring method of HD mold is based on the utilization of glass fibers into which several fiber Bragg gratings (FBG) have been incorporated in a cascaded manner. Each grating in a fiber serves as a temperature-measuring point, has a defined grating spacing and reflects only the characteristic wavelength of the initiated light spectrum. The optical measuring principle is completely insensitive to electromagnetic fields. The sensors are a permanent component of the mold plate and remain there in a well-protected condition during the entire period of operation of the mold.

By permanently monitoring the heat transmission in the mold, exact tracking of nonsteady-state process sequences becomes possible. The intensive insight into process stability under steady-state process conditions offers important points of departure for finding the causes for near-surface defects and for defining suitable counter-measures. By making a comparison with quality data on the intermediate or end product, it will be possible in the future to examine the effects of meniscus fluctuations on the morphology of oscillation marks and on the formation of longitudinal and transverse depressions or cracks. Based on fiber optical measurement several models were developed.

\subsection{Breakout Prevention Assist}

The formation of stickers constitutes a considerable risk for the casting process. Stickers often lead to breakouts and therefore have severe consequences for the casting machine, the product and productivity. Thanks to the number of optical fibers and their positions, the formation of stickers is detected at an early stage. Situations which could not be handled in the past and which in the worst case would lead to breakouts are now safely detected and rectified [1]. Better signal dynamics of fiber optics gains up to 7 seconds in comparison to thermocouples, Fig. 1. 

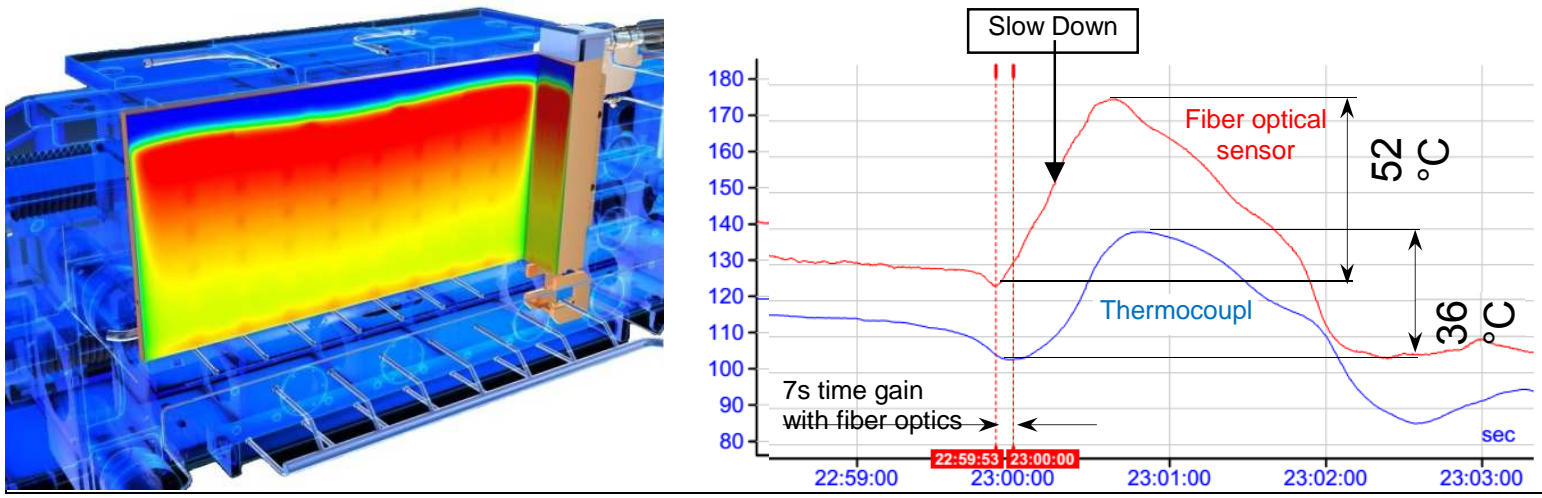

Fig. 1 Comparison signal dynamics fiber optics vs. thermocouples under casting conditions

\subsection{Longitudinal Crack Assist}

Longitudinal cracks are known from visual inspection of the cold slab. The visual inspections correlate with cases of abnormal temperature drop in measuring recordings in the mold.

The appearance of longitudinal cracks is a harmful product defect. In some rare cases the strand shell is weakened so much by the longitudinal cracks that the thermo-mechanical stress during casting opens up the crack and results in a breakout. To meet the required quality demands, the process data and the longitudinal crack defects will be monitored and identified within the mold.

All known crack types are accompanied by a local characteristic disturbance in the heat extraction in the mold. Small longitudinal crack occur with a length of 4 centimeters (refer to right sketch of Fig. 2, part of the visualization screen of HD mold) or larger on the broad sides of the cast product.

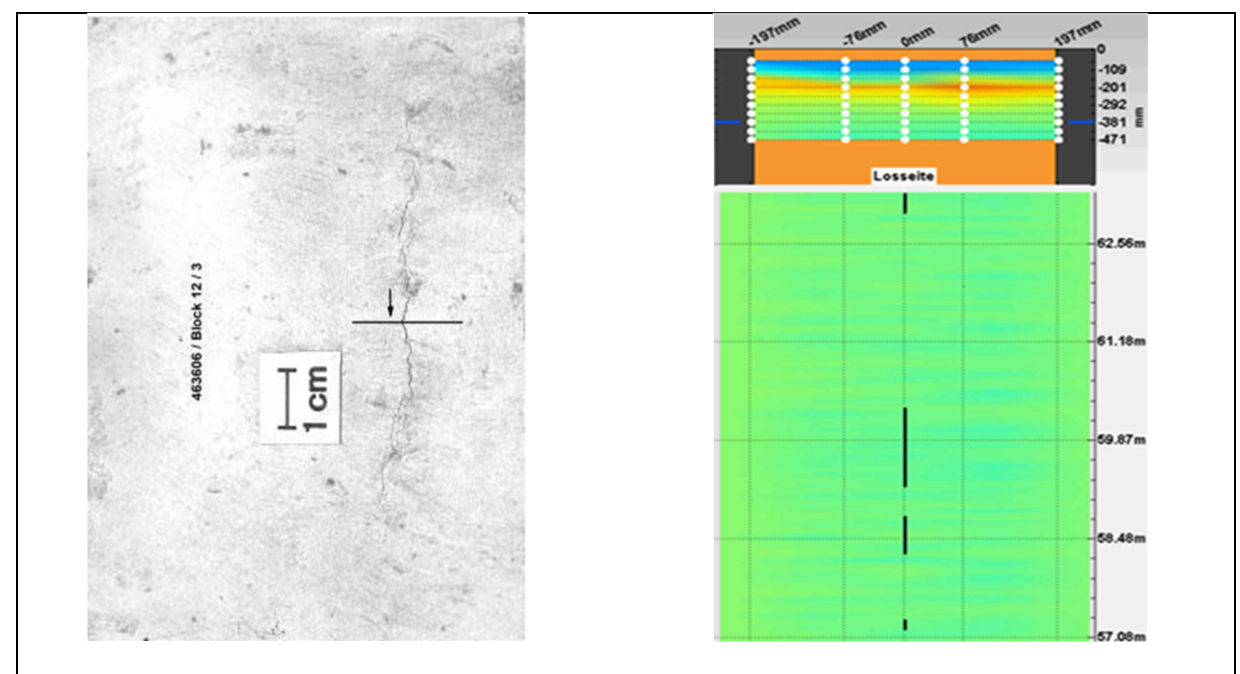

Fig. 2 Longitudinal crack $4 \mathrm{~cm}$ size

\subsection{Solidification Assist}

A computational model of heat transfer, solidification and phase transformation was applied to interpret the temperature measurement online. Fine measurement resolution gives a closer insight into primary strand shell formation in the mold. Precise calculation of local strand shell thickness and thermal resistance of mold flux 
was realized. This fundamental information is essential for the comparison of mold thermal conditions for different steel grades and mold powders $[2,3]$.

\subsection{Mold level Assist}

The causes of the heat transfer disturbances are often directly related to the addition of casting powder. Casting powder charging in intermittent mode causes in sudden liquid steel level drops directly after addition and it's upwards displacement with continuous consumption of casting powder. Based on temperature distribution, automatic mold powder feeding and wave contour control was made possible [4].

\subsection{Taper Assist}

The quality of continuously cast products in the edge zone between the broad and the narrow faces also depends on the taper. Longitudinal cracks in the edge zone may formed by an excessive mold taper; here, the pressure of the narrow faces on the strand produces a depression at the edge of the broad face, Fig. 3.

The contact behavior of the narrow faces from the meniscus to the mold outlet defines the heat dissipation and thus the formation of the strand shell. Narrow faces equipped with optical fibers across their entire height facilitate the measurement of heat flux densities. Here, the local heat fluxes across the narrow face height are determined - resolved to the exact location. The measurements immediately provide information on deviations in the contact behavior of the narrow faces and thus form the basis for the readjustment of the narrow faces during the casting process.

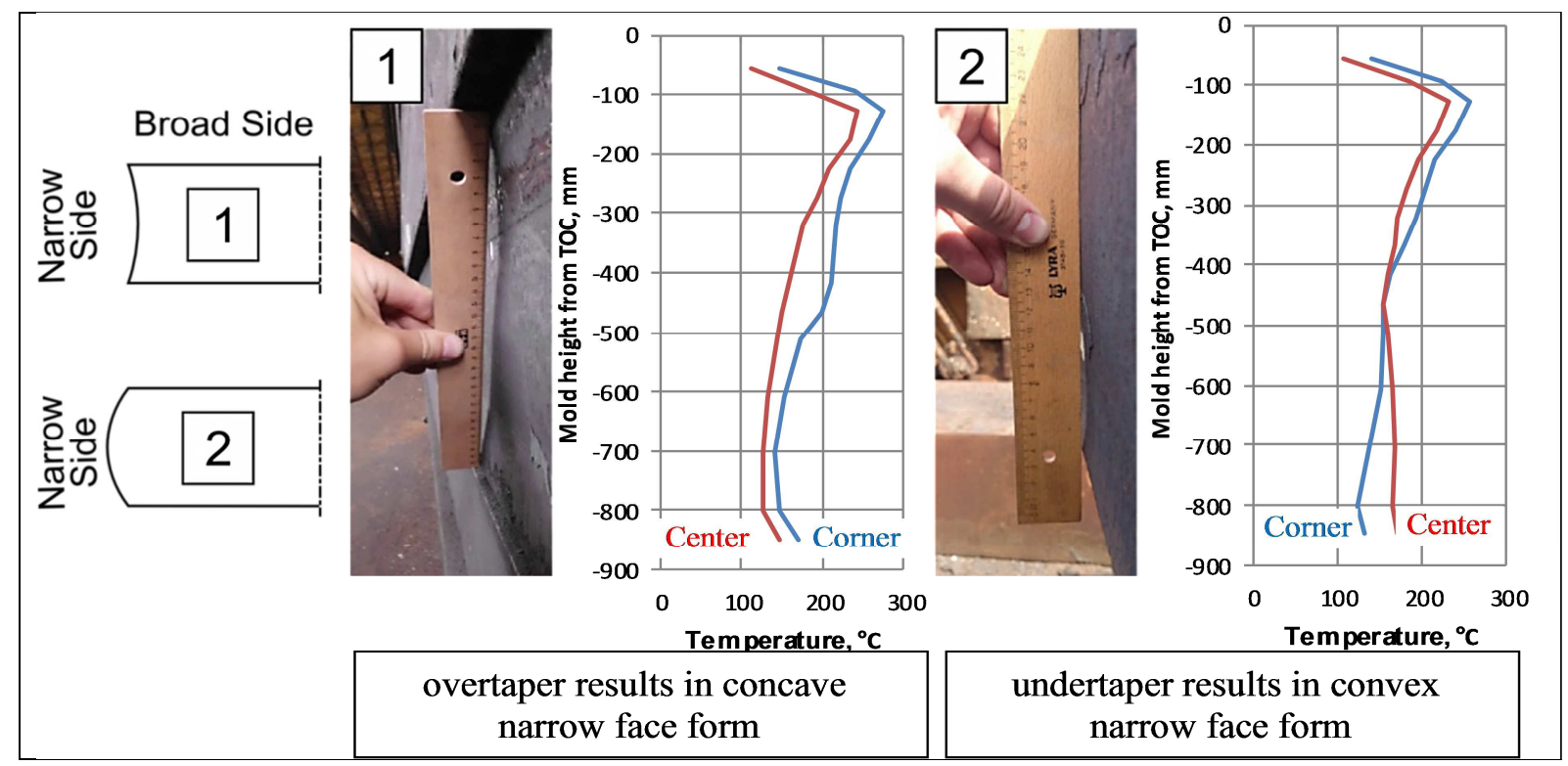

Fig. 3 Non-optimal taper adjustment detected with HD mold taper assistant under casting conditions

\section{SLAB CORNER TEMPERATURE WITH THE HD SPRAY SECONDARY COOLING CONCEPT}

A wide range of steel grades of different slab widths in modern slab casters requires maximum flexibility in the secondary cooling. Micro-alloyed steel grades in the peritectic and medium carbon range require a certain temperature range above or below the so called ductility trough in the slab straightening area in order to avoid 
corner or gutter cracking. Cooling strategies for ultra-soft and ultra-hard slab edge cooling are known. Both strategies require edge temperature control independently of the slab width. For each plant owner, the design of the secondary cooling section is a compromise between investment price, setting range and maintenance expenditure. SMS group offers various secondary cooling concepts depending on process conditions. A new secondary cooling concept for refined edge cooling is presented [5]. Requiring only two controlled water loops over the width, the new concept can realize five and even more cooling zones along the width direction.

\subsection{Advanced Concept of Width-Dependent Secondary Cooling}

If the water spray density cannot be variably adjusted over the width, the nozzles will spray onto the narrow face of the slab, since less than half of the spraying width of the nozzles covers the slab. As a result, the slab edges will become overcooled. To prevent the edges from becoming overcooled, the affected nozzles have to be switched off. Furthermore, the next row of nozzles must be supplied with a smaller quantity of water.

For comparison of the various cooling concepts, simulations of the peritectic steel grade for high-strength tube grades were performed by using the mathematical solidification model DSC ${ }^{\circledR}$ from SMS group, Fig. 4 and Fig. 5.

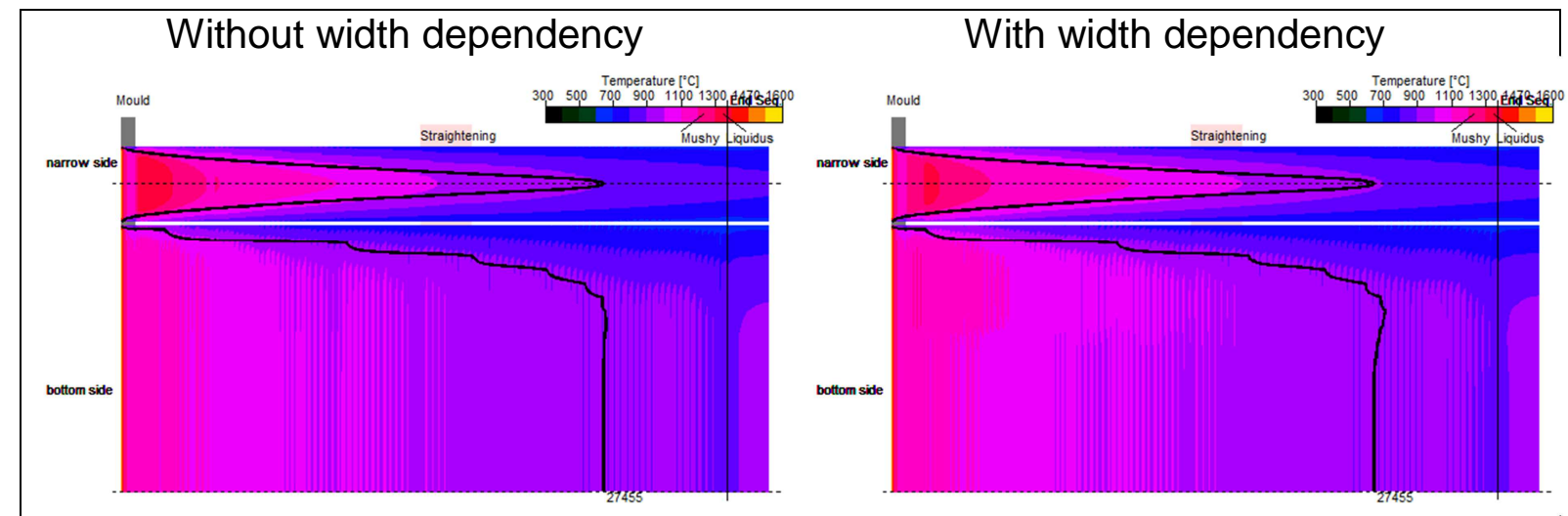

Fig. 4 Isotherms on surface

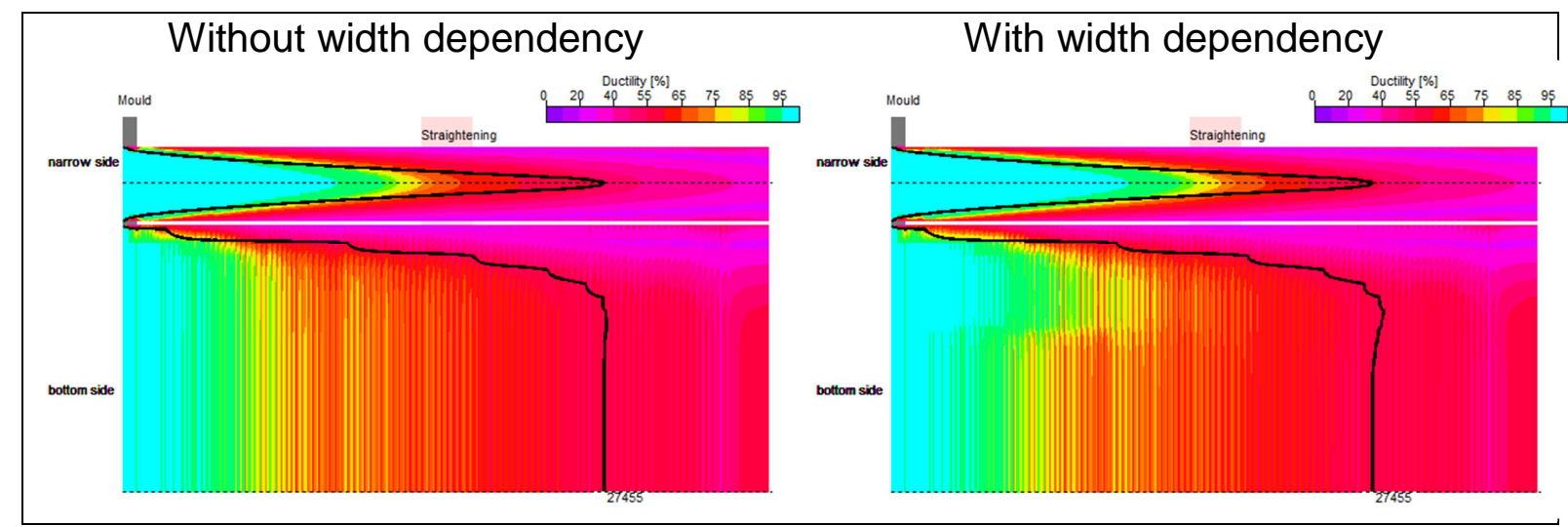

Fig. 5 Ductility on surface

Furthermore, a continuous slab caster for $250 \mathrm{~mm}$ thick slabs with a radius of $11.5 \mathrm{~m}$ and a metallurgical length of $34 \mathrm{~m}$ was simulated. The following process parameters were stipulated:

Casting speed $-1.0 \mathrm{~m} / \mathrm{min}$, Slab width $1,800 \mathrm{~mm}$ 
Results of the HD spray, width-dependent secondary cooling are shown in the following Fig. 6. A ductility value of $50 \%$ was assumed as the criteria for the occurrence of cracks.

By applying HD spray, the width-dependent secondary cooling concept, the edge temperature in the straightening area was increased to around $50^{\circ} \mathrm{C}$ in comparison with a design not dependent on width. This decreases the risk of crack formation dramatically.

\subsection{Two Control Loops - A Large Width Range}

Two separate water control loops and a compressed-air control loop, operated by a pneumatic unit, enable fully continuous homogeneous cooling of slabs in the range of, for example, $1,100 \mathrm{~mm}$ right up to $2,600 \mathrm{~mm}$ width, depending on the switching position. A suitable switching position of the valves for twin-slab cooling was also implemented. The stipulated volumetric flow of water is distributed uniformly over the nozzles active in the circuit. During this, the first control loop is always operated with a comparatively low volumetric flow in order to counteract any overcooling in the edge areas, Fig. 6.

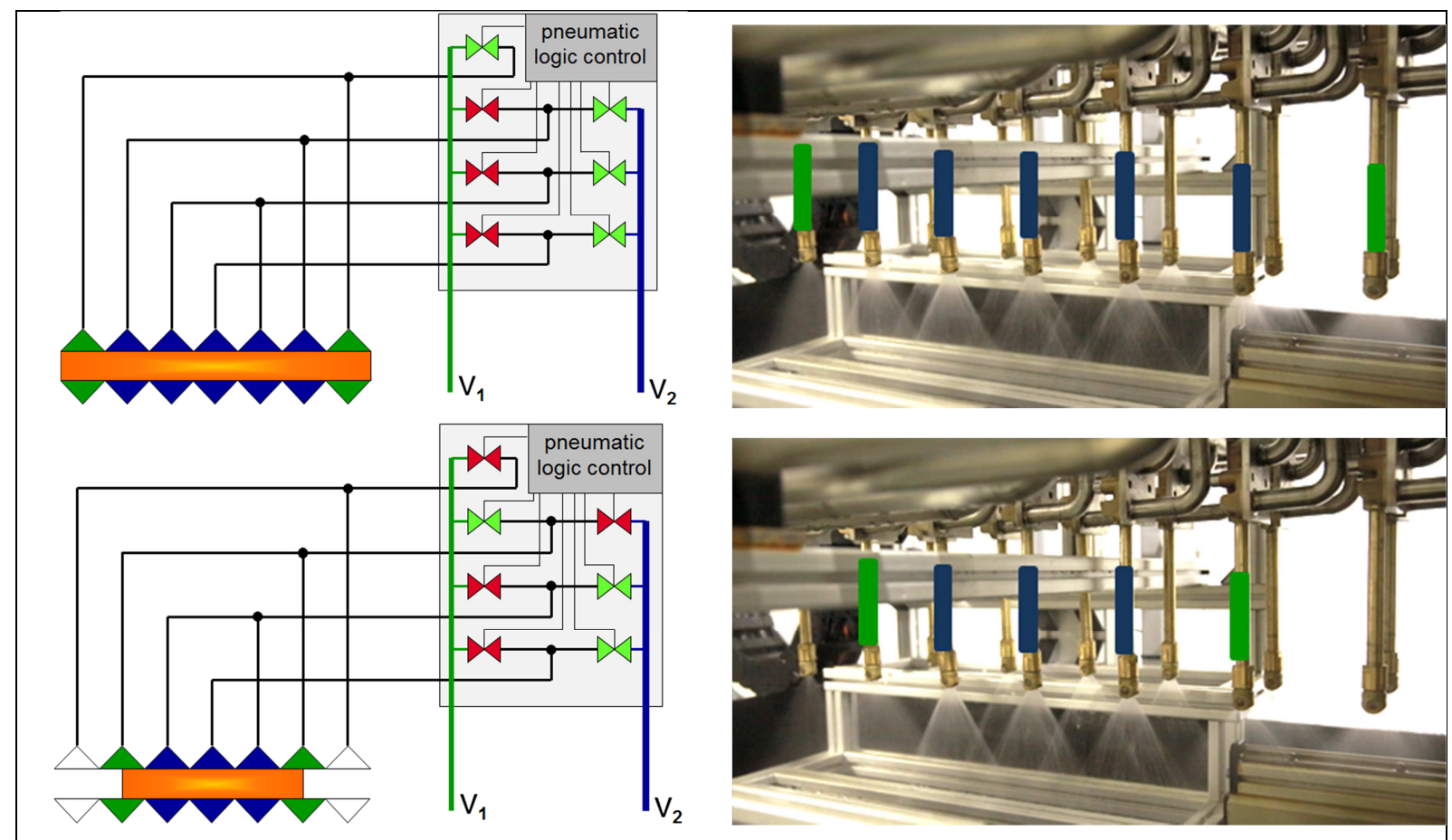

Fig. 6 Switching positions for different widths

\subsection{The Components for HD Spray}

The most important module is a water-distributing block with shut-off valves, Fig. 7 . The large number of possible combinations of active and deactivated nozzles is made possible by specifically controlled switching positions of the seven shut-off valves with very high switching power. By activating various pressure stages, the upstream pneumatic unit together with proportional pressure controller performs the switching on and off of the individual shut-off valves. This makes it possible to cool different slab widths. Finally, in the water-distributing block, the volumetric flow of the supplied water is distributed uniformly among the piping of the two water control 
loops. In order to withstand the adverse conditions during the casting process, the HD spray components are of robust design and made from stainless steel. A stainless-steel hood offers additional protection and guarantees maximum service life.

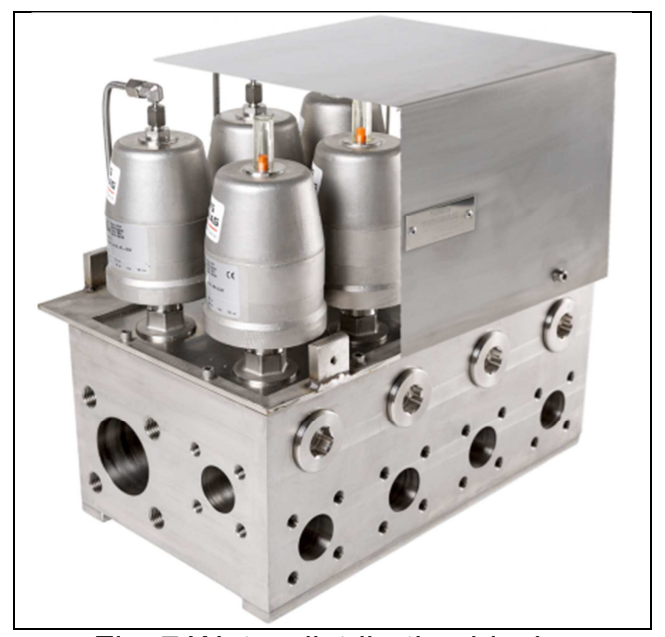

Fig. 7 Water-distributing block

\section{AT THE FOREFRONT - HD SIZE TO OPTIMIZE THE SLAB GEOMETRY ONLINE}

$\mathrm{HD}$ size is operated immediately downstream of the caster and upstream of the torch cutter and therefore in the position to survey the strand which has just fully solidified. The geometry of the cast slabs, the size (edges) and deformations are detected online and correlated with the relevant process parameters. Deviations from the specified size are recognized and can be corrected at an early stage. For a given casting mold dimension the cold slab dimensions like width and thickness depend on casting parameters like steel grade, casting speed, secondary cooling and soft reduction.

As it is difficult to model the described phenomena in a physical process model usually statistical model approaches are applied for the online process control. Due to limitations in model accuracy nowadays the slabs are often cast wider than necessary which requires later a slab sizing press treatment. If the slab sizing is not applicable due to quality restrictions the hot rolled coiled strip will have excess width. Measuring the real slab dimensions already in the continuous caster enables a better adaption of caster process control settings meeting the final quality assessment more accurately.

Therefore, any measurement system is exposed to the harsh environment in the vicinity of a caster. High temperature radiation, dust, scale as well as water vapor distort the measurement results strongly and have to be overcome. Caster-specific data as well as steel grades to be cast, production rate and the slab geometry benefit from the findings to achieve a higher yield and an enhanced quality of the produced slabs.

HD size can be set up as perfect stand-alone solution for any caster regardless of the existing automation landscape. 


\section{HD SCAN - ULTRASONIC QUALITY CONTROL REPLACES MACRO-ETCHING}

A new method for the inspection of internal quality of cast products has been developed by SMS group. This is based on ultrasonic testing of internal flaws and macrostructure. The simple and high informative ultrasonic technique is beneficial for the optimization of cast parameters, such as secondary cooling, soft reduction or mold and strand stirring.

The ultrasonic quality evaluation system offers reliable and objective quality results fully independent of etching parameters and subjective quality operator impressions. Therefore the conventional etching method may be completely replaced and the number of etching tests will significantly be reduced. The inner quality of other metallurgical products can also be analyzed with HD scan.

\subsection{Measurement System}

The system consists of a water basin to place the samples to be analyzed. On top a three dimensional adjusting device is carrying the ultrasonic sensor head. The ultrasonic sensor head consists of a flaw and a backwall sensor. The electronic unit initiates and measures the ultrasonic signal in accordance with the position and the movement of the sensor head.

The measurement technique is rather simple - after sample charging and short identification the automated measurement begins. Sophisticated software automatically evaluates quality parameters on the basis of measurement results. The size of the instrument is optimized according to sample geometry and aimed throughput. For the development of the HD scan we have chosen a size of $1,5 \times 1,5$ $\mathrm{m}^{2}$ for the basin for up to nine slab samples per charge, Fig. 8. This leads to a performance of up to 22 slab samples per day.

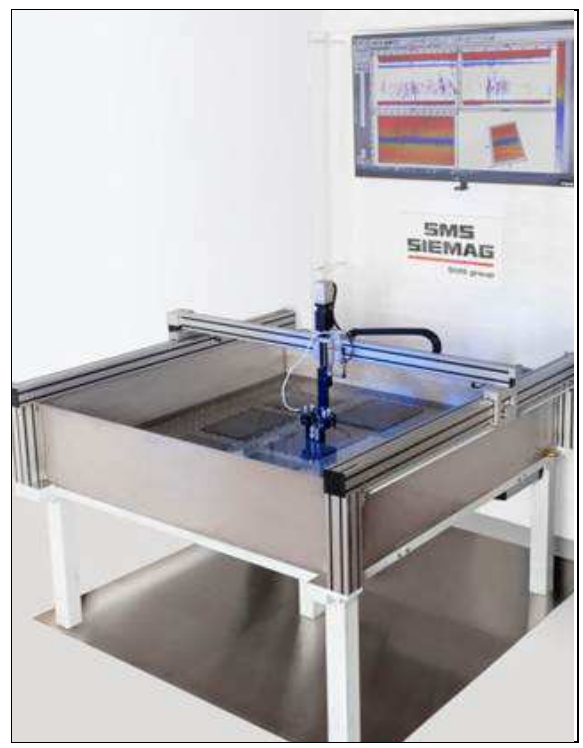

Fig. 8 HD scan

\subsection{Deeper Look Inside with No Additional Effort}

The HD scan analyzes samples as they are used for usual etching procedure. These are cross-sections of slabs or blooms as well as any other casted product perpendicular or parallel to casting direction. 
The surface requirements are not as demanding as they are for conventional etching laboratories. No grinding is needed; roughly milled (as scarfed) surface quality is sufficient and even rust on the surface does not influence the quality of the ultrasonic testing result. Thus with HD scan the preliminary work is reduced and saves time and effort of the whole quality control procedure.

The output of the ultrasonic measurement is more informative compared to etching image as not only one layer (sample surface in case of etching), but a volume of a sample is measured and represented in 3D-view with a possibility to evaluate different layers in the depth of the sample. This feature was shown to be very advantageous, as the sample surface, which is chosen by chance, is not always a reliable representation of product quality.

For example in case of $\mathrm{V}$-segregations in cast product a picture of segregation distribution in the sample differs much with the sample depth. In such case the quality of the product can be stated too good or too bad. Therefore a threedimensional view inside the sample with ultrasonic provides a more convincing and representative result. Furthermore 3D-representation is also beneficial for determination of the direction of flaw propagation in the material (for example cracks) in order to trace their origin and elaborate countermeasures. Hence ultrasonic technique also assists quality assurance.

\subsection{Environment}

The HD scan with ultrasonic technology is clean and safe with no risk for health and environment. No aggressive acids are used, no expensive safety precautions and equipment is necessary. The recycling of used acids no longer is required.

\subsection{Macrostructure}

Additionally macrostructure of cast samples can be visualized with HD scan even for samples did not succeed by macro-etching procedure. This can help in the optimization of cast parameters for well-known and for new steel grades. Areas of equiaxed and columnar crystallization can be distinguished and quantitatively determined. Fig. 9 shows a complete slab cross section consisting of 6 ultrasonic pictures.

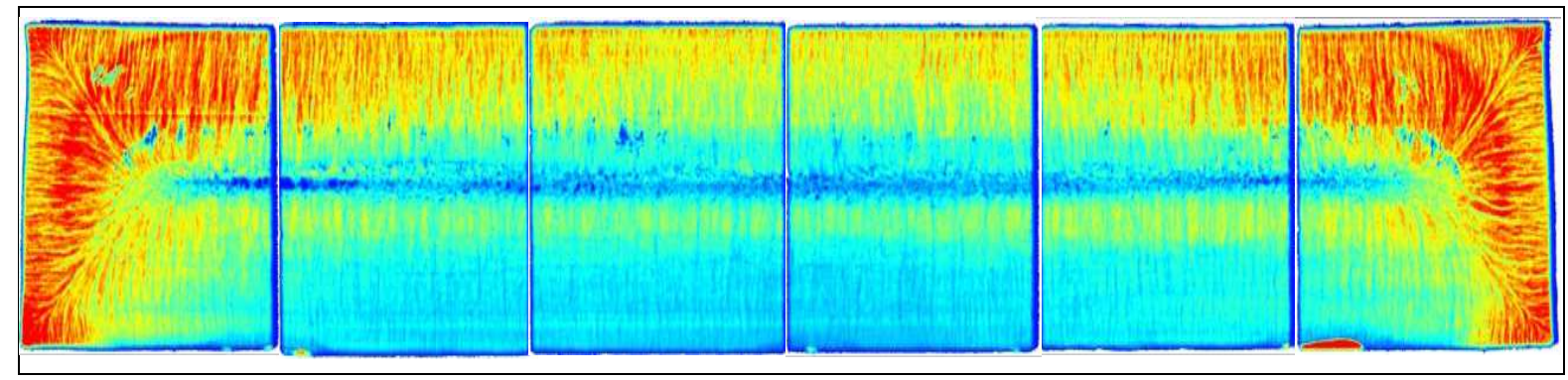

Fig. 9 Ultrasonic scan result of a complete slab cross section

\subsection{Automatic Classification}

The quality evaluation is done automatically and based on clear statistical rules derived from internal knowhow of SMS group and results from external experts. Size and distribution of the segregation are used for a segregation classification. Customer specific product classification rules can be implemented additionally, which 
gives the opportunity in comparing results of etching and HD scan. Automatic defect determination and classification was verified by comparing them with macro-etching classification results on more than 100 samples of different cast products. Cracks statistics are displayed including crack size, position, distances, etc. as well as the determination of the narrow side profile contour is realized.

\section{CONCLUSIONS}

With this paper we present you an outlook to desired technologies. The thin, medium and thick slabs produced on continuous casters from SMS group form the basis for superior steel products. In addition to the world's most extensive portfolio of continuous casting plants we create the HD family to improve slab quality sustainable. HD stands for High Definition or the possibility to get a more detailed inside view into the process with the option to act.

The High Definition family - HD mold, HD spray, HD LASr, HD size and HD scan demonstrates the persistence and sincerity we develop continuous casting for future challenges. This means a step ahead for steel producers for an enhanced so called highly defined product quality as well as an increased productivity. Two examples:

1) The first time ever: we introduce optical fiber measuring system (faser Bragg system) into industrial continuous casting mold. And the convincing results are an increased amount of measuring points for a deeper look inside the process. We offer the highest flexibility of position of measuring points. We provide the best available system to detect defects for further process refinement.

2) Ultrasonic examination is well known standard technology - e.g. from the doctor's and for example to have a healthy babe. We have a newborn system too, it's clean and secure! SMS group transferred ultrasonic to metallurgy and enhanced it by especially developed evaluation software. This is the one and only coherent system for such purpose in the world of metals. You will be amazed about the internal quality aspects of your slabs which will lead you to implementation of new systems and technologies of SMS group.

\section{REFERENCES}

1 Wans, Jochen; Lieftucht, Dirk; Geerkens, Christian; Arzberger Matthias: HD mold caster assistance system to increase product quality. AISTech 2013, Pittsburgh, PA, US, May 6-9, 2013, Proceedings 2:1385-1392

2 Krasilnikov A., Lieftucht D., Reifferscheid M., Hovestädt E., Schramm T., Kirsch D., Scheller P.R.: "Online shell thickness calculation operating in a new fiber optical based Mould Monitoring System": 5th International Congress on the Science and Technology of Steelmaking, Dresden, Germany, 1-3 October 2012

3 Krasilnikov, Artemy; Lieftucht, Dirk; Schlüter, Jochen; Reifferscheid, Markus: „Local heat transfer through mold flux film under casting conditions", 4th VDEH-CSM Metallurgical Seminar, Düsseldorf, Germany, 20-21 October 2014, Proceedings pp.217-224

4 Lieftucht, Dirk; Reifferscheid, Markus; Schramm, Thomas; Krasilnikov, Artemy, Kirsch, Dieter: HD mold - a new fiber- optic-based mold monitoring system. Iron \& Steel Technology, Dec. 2013, pp. 87-95

5 Krasilnikov, Artemy; Lieftucht, Dirk; Reifferscheid, Markus: „Slab corner temperature with the "HD spray" secondary cooling concept", European Continuous Casting Conference, Graz; 06/2014, Proceedings pp.1211-1219 